\title{
On the Estimation of Parameter of Weighted Sums of Exponential Distribution
}

\author{
N. Abbasi, A. Namju, and N. Safari \\ Department of Statistics, Payame Noor University, Tehran 19395-4697, Iran \\ Correspondence should be addressed to N. Abbasi; nargesabba30@yahoo.com
}

Received 4 November 2013; Accepted 19 December 2013; Published 4 March 2014

Academic Editors: S. Deng and W. Wang

Copyright (C) 2014 N. Abbasi et al. This is an open access article distributed under the Creative Commons Attribution License, which permits unrestricted use, distribution, and reproduction in any medium, provided the original work is properly cited.

\begin{abstract}
The random variable $Z_{n, \alpha}=Y_{1}+2^{\alpha} Y_{2}+\cdots+n^{\alpha} Y_{n}$, with $\alpha \in \mathbb{R}$ and $Y_{1}, Y_{2}, \ldots$ being independent exponentially distributed random variables with mean one, is considered. Van Leeuwaarden and Temme (2011) attempted to determine good approximation of the distribution of $Z_{n, \alpha}$. The main problem is estimating the parameter $\alpha$ that has the main state in applicable research. In this paper we show that estimating the parameter $\alpha$ by using the relation between $\alpha$ and mode is available. The mean square error values are obtained for estimating $\alpha$ by mode, moment method, and maximum likelihood method.
\end{abstract}

\section{Introduction}

The exponential distribution is one of the most applicable distributions in survival models and phenomena with memoryless property. Random size sample from the exponential distribution and considering the discrete distribution are the basis for creating new distributions. Proschan [1] showed that combinations distributions with constant failure rate have decreasing hazard function. So, in recent years, new distributions are introduced based on generalization and correction of the exponential distribution. For example, Adamidis et al. [2] find a new bivariate distribution, exponential-geometric distribution, with decreasing failure rate that is used in survival models. This distribution combines with exponential and geometric distributions. In the case that parameter of exponential distribution has decreasing-increasing failure rate the new distribution is called exponential-weibull that in fact is a generalized of exponential-geometric distribution introduced in 2010 by Silva et al. [3]. In all these studies trough estimating the parameters algorithm EM has been used.

Consider $Z_{n, \alpha}=Y_{1}+2^{\alpha} Y_{2}+\cdots+n^{\alpha} Y_{n}$ as the random variable with $\alpha \in \mathbb{R}$ and $Y_{1}, Y_{2}, \ldots$ being independent exponentially distributed random variables with mean one. In special case distribution function of the random variable
$Z_{n, \alpha}$ is a simple alternating series. For the case $\alpha=1$ accurs in various contexts, such as linear combinations of order statistics, noise in radio receivers, and run models, Kingman and Volkov [4]. For all $\alpha \geq-1 / 2$ the random variable $Z_{n, \alpha}$ follows the central limit theorem and leads to random variable normal standard. The result of normal approach of $P\left(Z_{n, \alpha}<x\right)$ would be useful for $n \rightarrow \infty$ in terms of X's, which are near to mean $Z_{n, \alpha}$. For $\alpha=-1$ the random variable $Z_{n, \alpha}$ is independent from $n$ and identity distribution and perfectly shows that the random variable has exponential distribution. One example of extreme value theory is that $Z_{n, \alpha}$ has Gumbel distribution as $n \rightarrow \infty$. Because $\alpha=-2$ the random variable $Z_{n, \alpha}$ is Kolmogorov distribution. In general case, the distribution function is a series with alternating sign that Van Leeuwarden and Temme [5] derived an approximation uniform expansion for $\mathbb{P}\left(Z_{n, \alpha}<\right.$ $x$ ) by applying an extended version of the saddle point method.

In this paper in order to estimate the parameter $\alpha$, first we use the exact density of linear combination of independent exponential distribution. Determining the state of distribution for different values of $\alpha$ would lead us to exact value of it. Some results show the simulations between distributions in this family. Also to reach the estimation of parameter $\alpha$ we suggest sample mode. In Section 1 we review 
the density function of $Z_{n, \alpha}$ and its characteristics. We will show mode and sum result of it in Section 2. For estimating mode there are numerous formula [6]. But here, we used the following formula:

$$
\mathrm{Mo}_{s}=L_{\mathrm{Mo}}+\frac{d_{1}}{d_{1}+d_{2}} c,
$$

where $L_{\text {Mo }}$ is a lower bound in mode class, $d_{1}$ and $d_{2}$ are absolute difference frequency of mode class before and after it, respectively, and $c$ is a length of mode class. In statistical table, mode class is a class that has high frequency. At the end, using trough mean square error we compare three estimators for $\alpha$, according the sample mode, the moment sample, and maximum likelihood estimator.

\section{Density of $Z_{n, \alpha}$}

The distribution function of the random variable $Z_{n, \alpha}$ is

$$
F_{n, \alpha}(x)=P\left(Z_{n, \alpha} \leq x\right)=1-\sum_{j=1}^{n} \frac{1}{(\mathscr{T}(n, \alpha, j))} \cdot \exp \left(-x j^{-\alpha}\right),
$$

where

$$
\mathscr{T}(n, \alpha, j)=\left(\prod_{k=1}^{j-1}\left(1-\left(\frac{k}{j}\right)^{\alpha}\right)\right)\left(\prod_{k=j+1}^{n}\left(1-\left(\frac{k}{j}\right)^{\alpha}\right)\right) .
$$

Then we can write

$$
\begin{array}{r}
f_{n, \alpha}(x) \\
=\sum_{j=1}^{n}\left(\frac{j^{-\alpha} \exp \left(-x j^{-\alpha}\right)}{\left(\prod_{k=1}^{j-1}\left(1-(k / j)^{\alpha}\right)\right)\left(\prod_{k=j+1}^{n}\left(1-(k / j)^{\alpha}\right)\right)}\right), \\
x>0 .
\end{array}
$$

In special case, for $n=2$ the distribution function is as follows:

$$
F_{2, \alpha}(x)=1-\frac{e^{-x}}{1-2^{\alpha}}-\frac{e^{-x 2^{-\alpha}}}{1-2^{-\alpha}}, \quad x>0, \alpha \in R .
$$

For $\alpha \leq-3$ and different values of $n$, the figures of distribution will be shown in Figures 1(a) and 1(b).

Based on the shape, it is clear that distributions are fair proximity to $f_{2, \alpha}$; that is,

$$
f_{n, \alpha} \approx f_{2, \alpha} ; \quad \forall n \geq 2, \alpha \leq-3 .
$$

This point can limit the estimating parameter $\alpha$, so we can estimate it accurately.

With some non complex calculation of $\partial f_{2, \alpha}(x) / \partial x=0$, we can obtain the mode:

$$
\text { Mode }=\frac{\ln \left(2^{-\alpha}\right)}{2^{-\alpha}-1} .
$$

TABLE 1: The distribution function values by considering different values $\alpha$.

\begin{tabular}{lcccccc}
\hline$\alpha$ & 1 & 2 & 3 & 4 & 5 & 12 \\
\hline$F_{2, \alpha}(\mathrm{Mo})$ & 0.25 & 0.214 & 0.165 & 0.119 & 0.078 & 0.001 \\
\hline
\end{tabular}

Next theorem is a good result to show the effect of $\alpha$ in distribution function based on mode value.

Theorem 1. If $h(\alpha)=F_{2, \alpha}($ Mode $)$, then $h(\alpha)=h(-\alpha)$.

Proof. Consider

$$
\begin{aligned}
h(\alpha)=1- & \frac{1}{1-2^{\alpha}} \exp \left(\frac{\ln \left(2^{\alpha}\right)}{2^{-\alpha}-1}\right) \\
& \left.+\frac{1}{\left(1-2^{-\alpha}\right)} \exp \left(2^{-\alpha} \frac{\ln \left(2^{\alpha}\right)}{2^{-\alpha}-1}\right)\right\} \\
= & 1-\left\{\frac{1}{1-2^{\alpha}} 2^{\alpha\left(1 /\left(2^{-\alpha}-1\right)\right)}+\frac{1}{1-2^{-\alpha}} 2^{\alpha\left(1 /\left(1-2^{\alpha}\right)\right)}\right\} \\
= & 1-\left\{\frac{1}{1-2^{\alpha}} 2^{\left(\alpha /\left(2^{-\alpha}-1\right)\right)}+\frac{1}{1-2^{-\alpha}} 2^{\left(-\alpha /\left(2^{\alpha}-1\right)\right)}\right\} .
\end{aligned}
$$

With considering $-\alpha$ instead of $\alpha$, we will have $h(\alpha)=h(-\alpha)$.

In Table 1 the distribution function values of $Z_{2, \alpha}$ in mode point by considering different values of $\alpha$ evaluated and showed in, as it's clearer, results repeated for negative points. Due to Theorem 1, correspondence negative values can be obtained.

Then we can see

$$
\lim _{\alpha \rightarrow \pm \infty} P\left(Z_{2, \alpha} \leq \text { Mode }\right)=0 .
$$

The formula in (7) showed that the relations of mode and mean with parameter $\alpha$ lead us to better estimator. Since we can estimate mode and mean trough sample data, we can find two estimators for parameter $\alpha$. Solution of the equation that known and unknown sides are sample and distribution mode, respectively, lead us to following answer:

$$
\widetilde{\alpha}_{\mathrm{Mo}}=\exp \left(- \text { lambert } W\left(-\frac{\text { Mode }}{e^{\text {Mode }}}\right)-\text { Mode }\right),
$$

where lambert $W(\cdot)$ is a defined function in MAPLE software.

The other estimator is obtained from the mean sample by MoM method and expectation of distribution. Let $\bar{Z}$ be the sample mean of $Z_{2, \alpha}$ 's; then

$$
\widetilde{\alpha}_{\mathrm{MoM}}=\frac{\ln (\bar{Z}-1)}{\ln (2)} .
$$

If $\bar{Z}<1$, the moment estimator is not achieved.

\section{Simulating and Comparing the Estimators}

The simulation that was performed with a sample size of 100 is considered to have been repeated 100 times. In Table 2, 


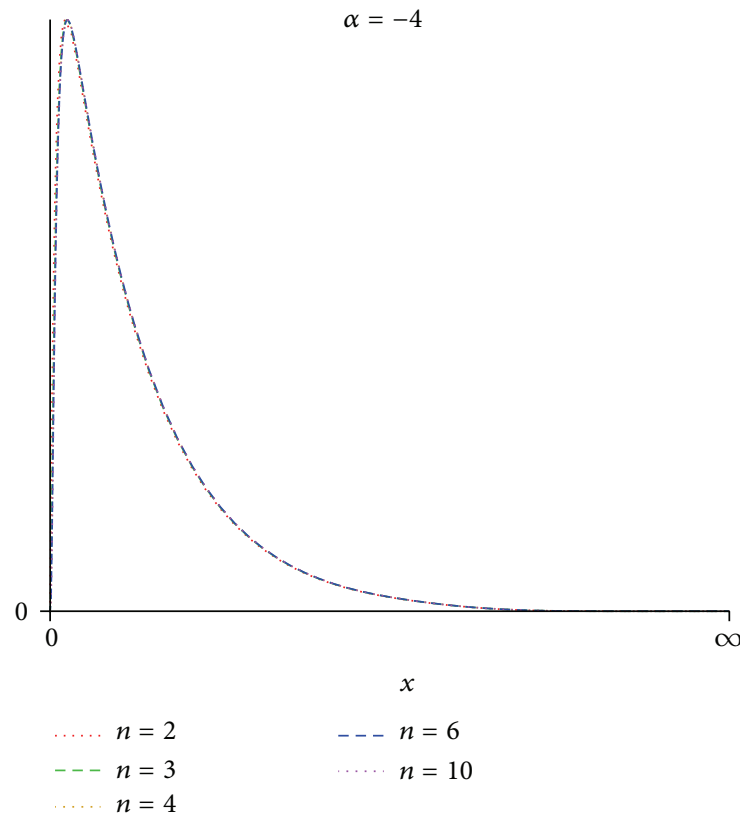

(a)

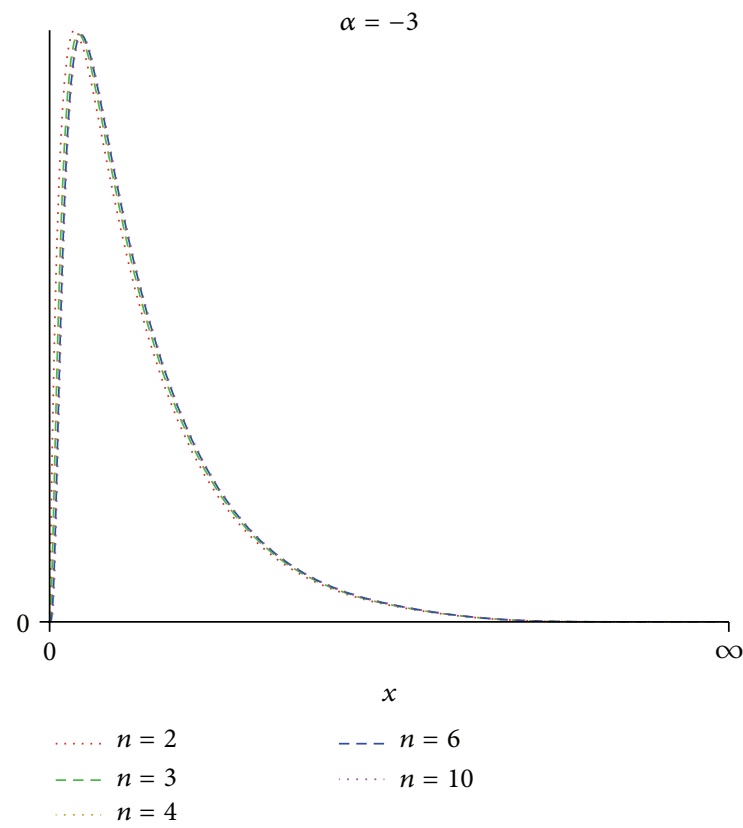

(b)

Figure 1: (a) The density of $Z_{n, \alpha}$ at $\alpha=-4$. (b) The density of $Z_{n, \alpha}$ at $\alpha=-3$.

TABLE 2: Comparing MSE values by three methods.

\begin{tabular}{lccc}
\hline$\alpha$ & MSE $\left(\widetilde{\alpha}_{\text {MoM }}\right)$ & MSE $\left(\widehat{\alpha}_{\text {ML }}\right)$ & MSE $\left(\widetilde{\alpha}_{\text {Mo }}\right)$ \\
\hline-3.0 & NAN & 0.006003296 & 0.021969958 \\
-2.7 & NAN & 0.005481582 & 0.026626967 \\
-2.5 & NAN & 0.005607409 & 0.018072630 \\
-2.2 & NAN & 0.008802720 & 0.018315792 \\
-2.1 & 0.016307740 & 0.006732829 & 0.028529943 \\
-2.0 & 0.016655870 & 0.006487885 & 0.014026310 \\
-1.5 & 0.009756532 & 0.006950620 & 0.013820559 \\
-1.0 & 0.009457660 & 0.007393859 & 0.021806514 \\
-0.5 & 0.004352376 & 0.003914091 & 0.106918369 \\
-0.1 & 0.006106967 & 0.003007703 & 0.186182937 \\
0.1 & 0.004722933 & 0.001817233 & 0.030854950 \\
0.5 & 0.003574097 & 0.003488361 & 0.031340420 \\
1.0 & 0.005017213 & 0.004843485 & 0.050798000 \\
1.5 & 0.004464924 & 0.004316831 & 0.050390390 \\
2.0 & 0.004734593 & 0.003770206 & 0.040783143 \\
3.0 & 0.006107225 & 0.005880179 & 0.659807293 \\
\hline
\end{tabular}

ignoring the problems, the phrase inside the log function that may be negative instead of some parameters $\alpha$, MSE values for all the three estimators method calculated. By numeric method, we compare three estimators, MLE, MoM, and estimator based on distribution mode.

As we expected, based on Table 2, MSE is more preferred than the two other estimators. While for some values $\alpha$ we cannot submit MoM estimator, MSE for sample mod estimator prefers MoM. Perhaps the use of other estimator modes improved alpha estimation.

\section{Conflict of Interests}

The authors declare that there is no conflict of interests regarding the publication of this paper.

\section{References}

[1] F. Proschan, "Theorical explanation of observed decreasing failure rate," Technimetrics, vol. 5, pp. 375-383, 1963.

[2] K. Adamidis, T. Dimitrakopoulou, and S. Loukas, "On an extension of the exponential-geometric distribution," Statistics and Probability Letters, vol. 73, no. 3, pp. 259-269, 2005.

[3] R. B. Silva, W. Barreto-Souza, and G. M. Cordeiro, "A new distribution with decreasing, increasing and upside-down bathtub failure rate," Computational Statistics and Data Analysis, vol. 54, no. 4, pp. 935-944, 2010.

[4] J. F. C. Kingman and S. E. Volkov, "Solution to the OK corral model via decoupling of Friedman's urn," Journal of Theoretical Probability, vol. 16, no. 1, pp. 267-276, 2003.

[5] J. S. H. Van Leeuwaarden and N. M. Temme, "A uniform asymptotic expansion for weighted sums of exponentials," Statistics and Probability Letters, vol. 81, no. 11, pp. 1571-1579, 2011.

[6] G. Ulf, "Some direct estimates of the mode," The Annals of Mathematical Statistics, vol. 36, pp. 131-138, 1965. 


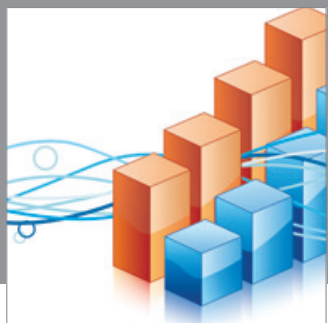

Advances in

Operations Research

mansans

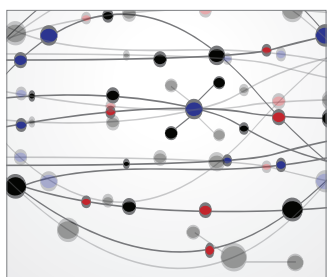

The Scientific World Journal
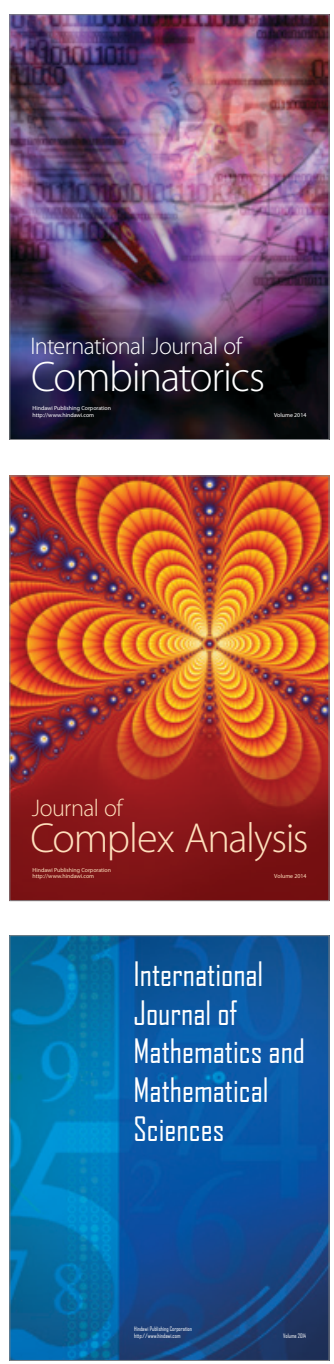
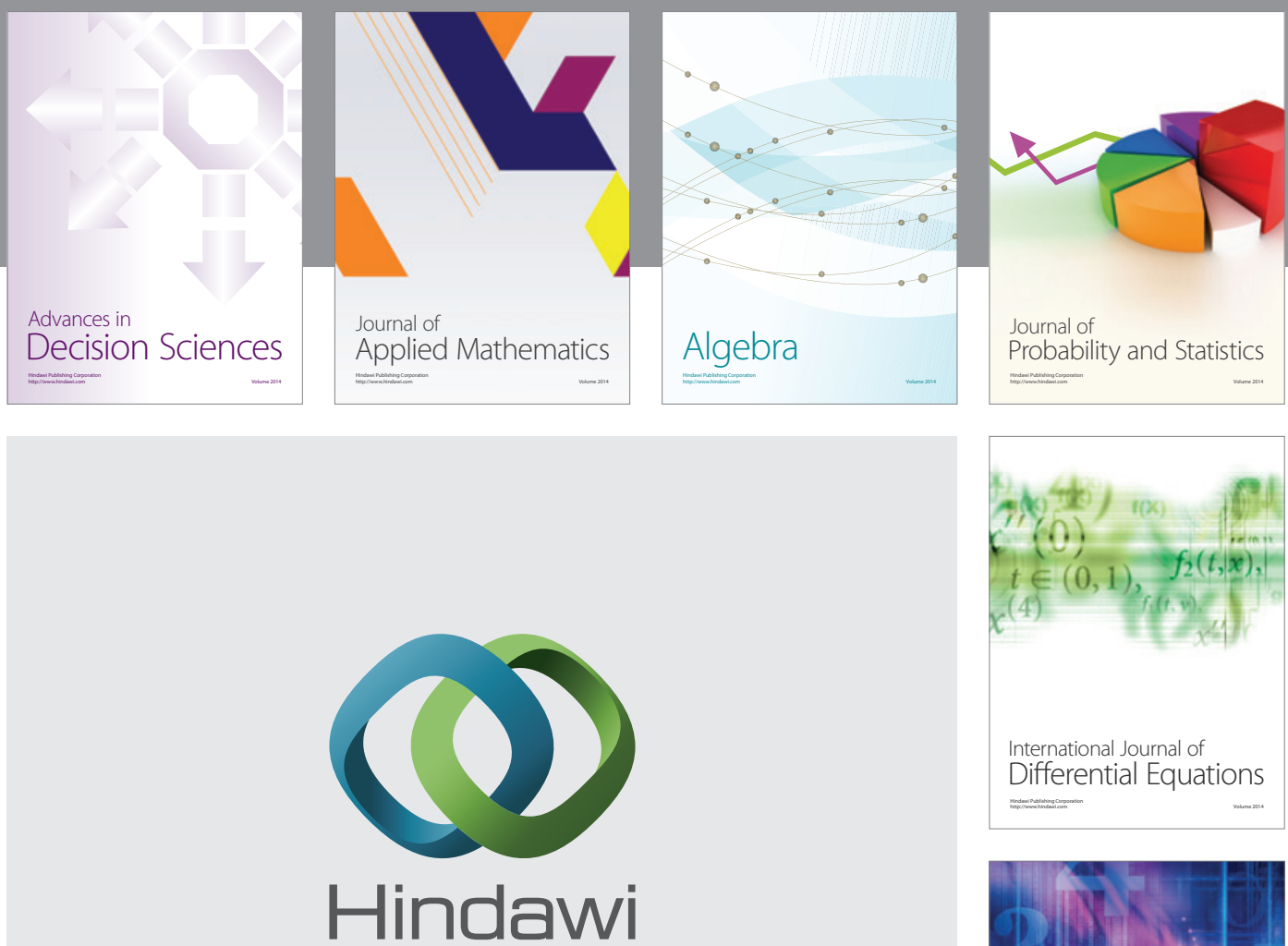

Submit your manuscripts at http://www.hindawi.com
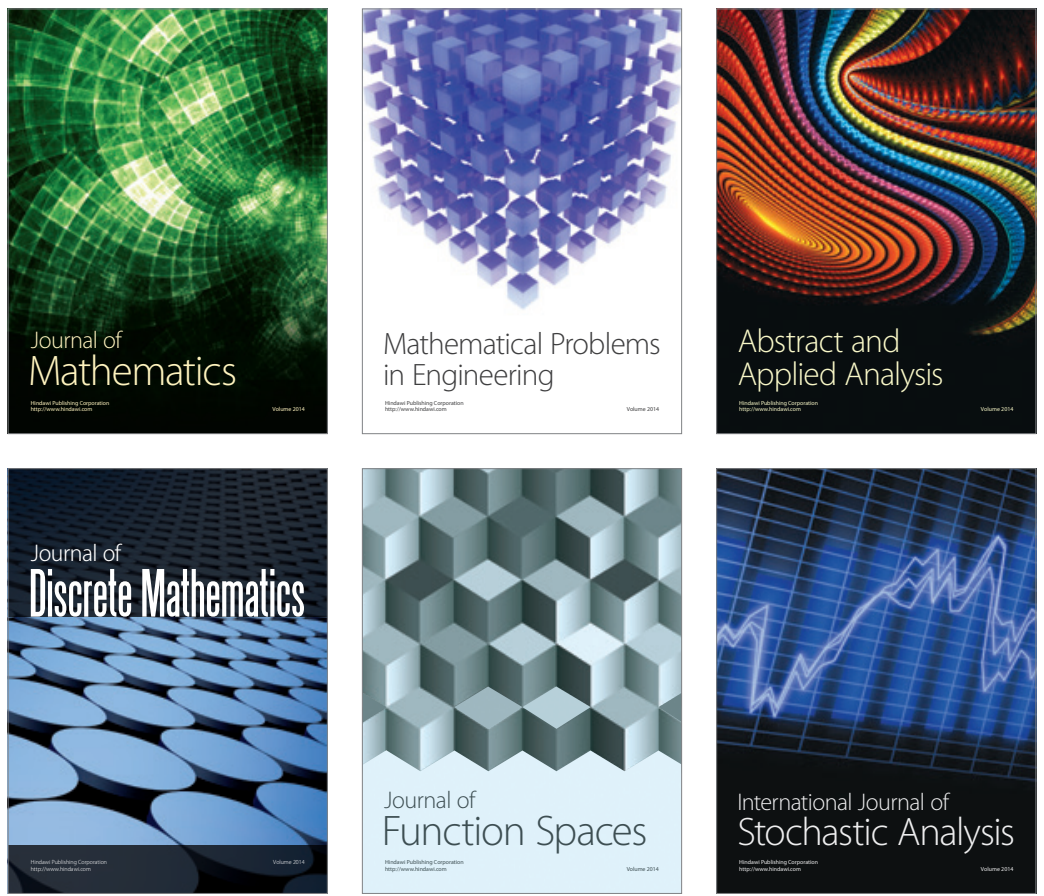

Journal of

Function Spaces

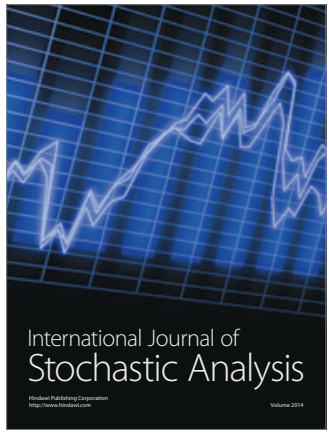

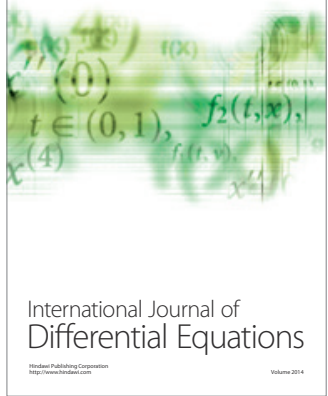
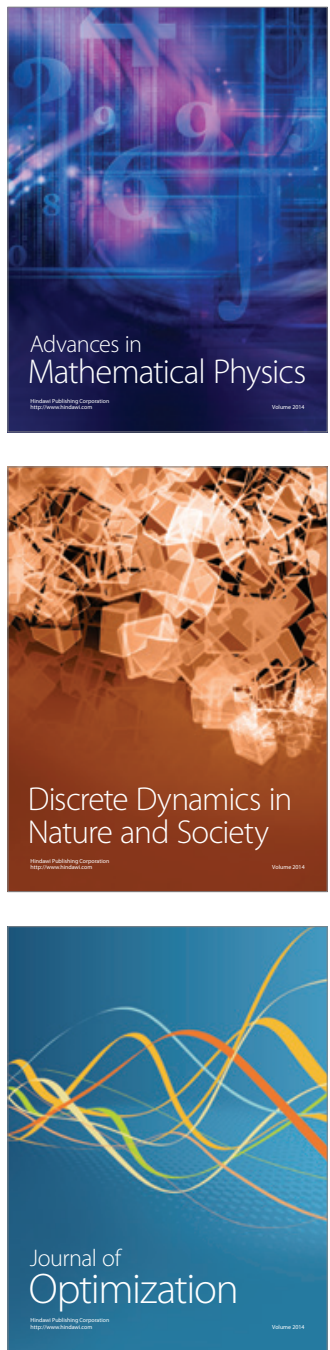